\title{
Hydrated Solid Forms of Theophylline and Caffeine Obtained by Mechanochemistry
}

\author{
Juan Saulo González-González*1, Oscar Zúñiga-Lemus ${ }^{1}$, María del \\ Carmen Hernández-Galindo ${ }^{1}$ \\ ${ }^{I}$ Instituto de Farmacobiología, Universidad de la Cañada. Carretera Teotitlán - San Antonio Nanahuatipán km \\ 1.7 s/n. Paraje Titlacuatitla. Teotitlán de Flores Magón, Oaxaca. México, C.P. 68540,
}

\begin{abstract}
Caffeine and theophylline are methyl-xantine drugs, which are affected by water leading to their hydrated forms. The purpose of this work was to prepare and characterize hydrated forms of caffeine and theophylline by mechanochemistry. The obtained products were characterized by infrared spectroscopy and Xray powder diffraction. The infrared spectra and diffractograms of the ground products were similar to the reported for caffeine and theophylline hydrates.
\end{abstract}

Keywords: Mechanochemistry, infrared spectroscopy, X-ray powder diffraction, caffeine, theophylline.

\section{INTRODUCTION}

Pharmaceutical Active Ingredients (API's) are the most important part in medicines. Most of API's are solids. Therefore, it is important to study their solid state properties. API's can be found as, salts, polymorphs, cocrystals and solvates [1].

Solvates are crystalline materials which incorporates a solvent molecule into the crystalline lattice. When the trapped solvent is water, then, is called hydrate [2]. Water can be incorporated into the crystalline lattice from the atmospheric humidity or in a step of the manufacturing process [3]. Hydration of an API can affect its biopharmaceutical properties, the manufacturing process and the stability of the final product [4].

Instrumental methods such as infrared spectroscopy, X-ray powder diffraction or single X-ray diffraction are used to identify solid forms of API's [5].

Caffeine and theophylline are methyl-xantine drugs which occur in natural products such coffee, tea and cocoa nuts. Caffeine is a central nervous system stimulant and theophylline is used for the treatment of asthma and chronic obstructive pulmonary disease [6,7]. Hydration of caffeine and theophylline is one of the problems which affect its physicochemical stability. Hydrated forms of caffeine and theophyline are obtained by recrystallization of the anhydrous forms in water $[8,9]$.

Mechanochemistry involves the use of mechanical forces (such as compression, shear or friction) to induce chemical transformations [10]. In pharmaceutical technology is applied to obtain polymorphs or pharmaceutical cocrystals [11]. In order to demonstrate the effect of water absorption over solid forms of API's, in laboratory sessions of pharmaceutical technology course, here we report a rapid method to obtain hydrated forms of theophylline and caffeine by mechanochemistry, and the characterization of the products by infrared spectroscopy and powder X-ray diffraction.

\subsection{Reagents and instrumentation}

\section{MATERIALS AND METHODS}

Caffeine and theophylline anhydrous were purchased from commercial sources and were used as received. Infrared (IR) spectra of solid samples of anhydrous caffeine and theophylline, and the hydrated products were obtained neat in a Bruker Tensor-27 spectrophotometer equipped with an attenuated total reflection (ATR) system (16 scans, spectral range $600-4000 \mathrm{~cm}^{-1}$, resolution $\left.4 \mathrm{~cm}^{-1}\right)$. X-ray powder diffraction data were collected on a PAN Analytical X-Pert PRO diffractometer with CuK $\alpha 1$ radiation $(\lambda=1.5405 \AA, 45$ $\mathrm{kV}, 40 \mathrm{~mA}$ ) from $2.02^{\circ}$ to $49.93^{\circ}$ in $2 \theta$. 


\subsection{Mechanochemistry}

Solid samples of caffeine and theophylline $(0.5 \mathrm{~g})$ and $0.5 \mathrm{ml}$ of distilled water were ground in a mortar with a pestle until complete 5 minutes of grinding time. Then, the powder was collected and stored in a glass vial.

\section{RESULTS}

\subsection{Infrared spectroscopy (IR)}

The IR spectra of the anhydrous caffeine and theophylline (Fig. 1) showed the characteristic stretching frequencies (Table 1) for the carbonyl $(\mathrm{C}=\mathrm{O})$, alkyl $\left(-\mathrm{CH}_{3}\right)$, amine $(\mathrm{N}-\mathrm{H}$, only for theophylline), functional groups. Incorporation of water into the crystalline lattice shifts the bands in the IR spectra of the hydrated theophylline and caffeine with respect to the anhydrous forms, as consequence of the hydrogen bonding pattern rearrangement. The O-H stretching frequency of water was observed at $3360 \mathrm{~cm}^{-1}$ for the theophylline hydrate and at $3357 \mathrm{~cm}^{-1}$ for the caffeine hydrate. The IR spectra of theophylline and caffeine hydrates were similar to the reported previously [12].

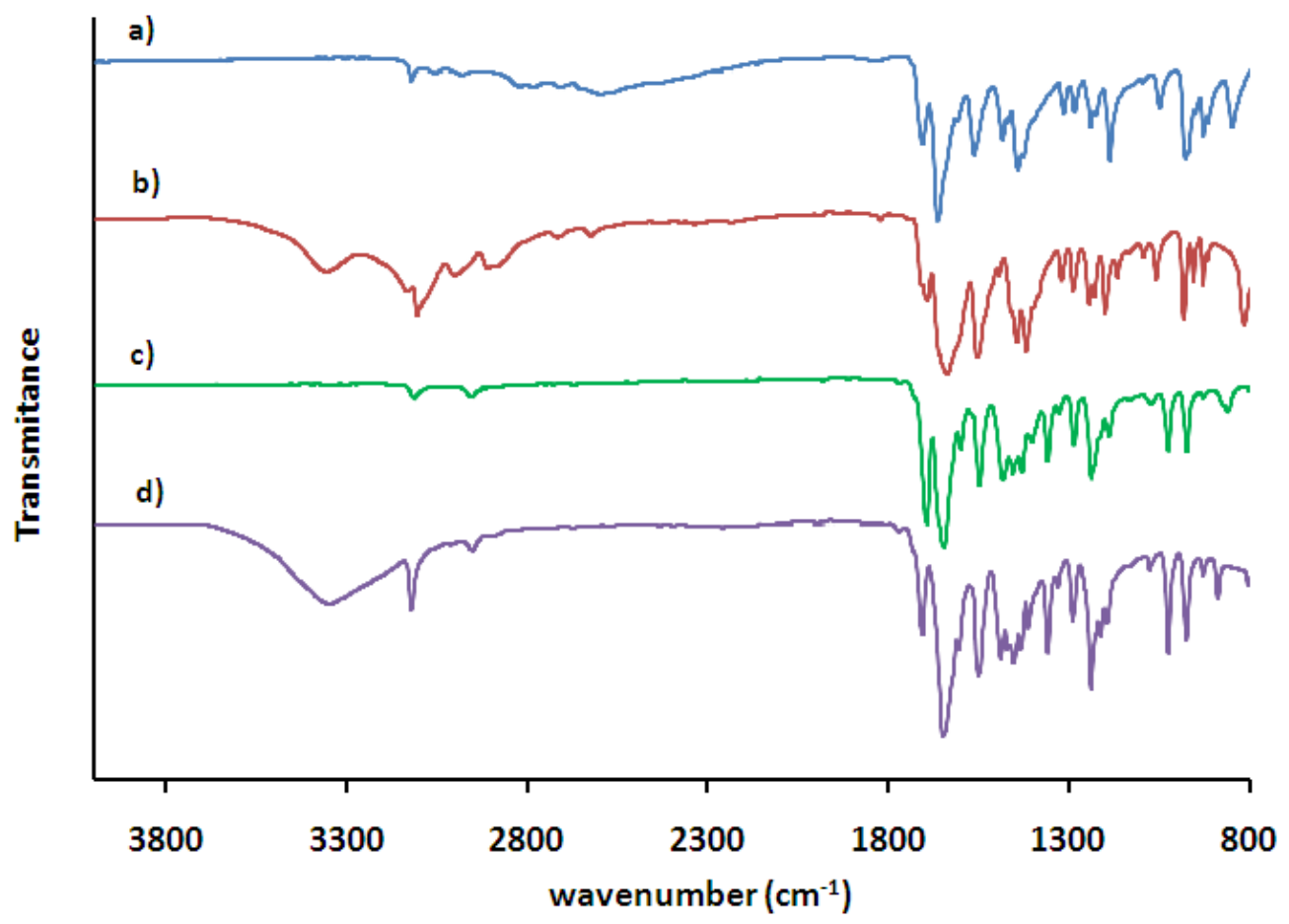

Figure 1. Infrared spectra of a) anhydrous theophylline, b) theophylline hydrate, c) anhydrous caffeine, and d) caffeine hydrate.

Table 1. Infrared frequencies of the functional groups of caffeine and theophylline, and the hydrates.

\begin{tabular}{|l|l|l|l|l|}
\hline & O-H & N-H & C-H (alkyl) & C=O \\
\hline TEO & --- & 3120 & 3055 & 1705,1662 \\
\hline TEO-hydrate & 3360 & 3131 & 3106 & $1708,1695,1642$ \\
\hline CAF & --- & --- & 3112 & 1694,1645 \\
\hline CAF-hydrate & 3357 & --- & 3121 & 1702,1650 \\
\hline
\end{tabular}

\subsection{X-ray powder diffraction (XRPD)}

In order to confirm the formation of the hydrated solid phase, XRPD study was carried out. The anhydrous theophylline showed a powder pattern similar to the polymorphic form II of theophylline [13], meanwhile the XRPD pattern of anhydrous caffeine belongs to the $\beta$-phase [8]. The XRPD patterns (Fig. 2 and Table 2) of the hydrated ground products showed the XRPD patterns of theophylline and caffeine hydrates reported previously [8,9], and also signals of residual anhydrous products. 


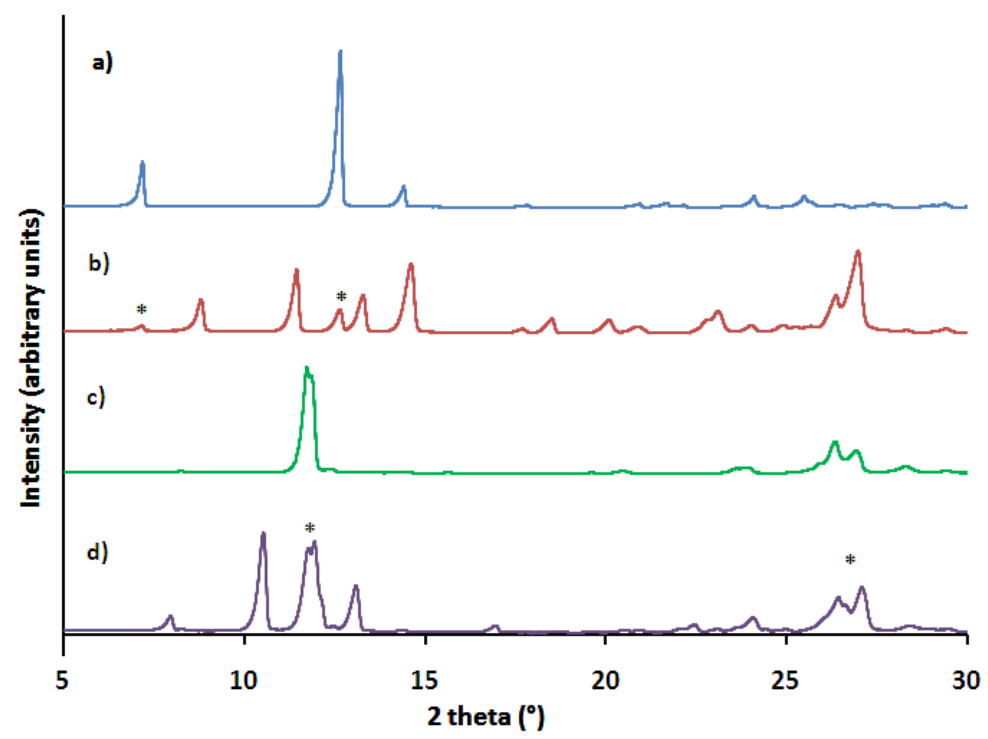

Figure 2. X-ray Powder Diffraction patterns of a) anhydrous theophylline, b) theophylline hydrate, c) anhydrous caffeine, and d) caffeine hydrate. (*) residual signals of anhydrous theophylline or anhydrous caffeine.

Table 2. $2 \theta$ signals of the anhydrous and hydrated products.

\begin{tabular}{|l|l|}
\hline & $\mathbf{2} \square$ signals $\left(^{\circ}\right)$ \\
\hline TEO & $7.18,12.66,14.39$ \\
\hline TEO-hydrate & $8.78,11.42,13.23,14.59,18.45,20.01,20.81,23.06,26.33,26.94$ \\
\hline CAF & $11.71,11.77,26.27,26.82$ \\
\hline CAF-hydrate & $7.90,10.50,13.05,16.81$ \\
\hline
\end{tabular}

\section{CONCLUSION}

It was obtained and characterized by IR spectroscopy and XRPD, the hydrated forms of theophylline and caffeine after 5 minutes of mechanochemical grinding with water, demonstrating the effect of water absorption over the API's.

\section{ACKNOWLEDGEMENTS}

To CONACYT (grant CB-2012 179674) and UNCA-CA-05 Farmacología Computacional for the support of this work.

\section{REFERENCES}

[1] N. Schultheiss, and A. Newman, Pharmaceutical Cocrystals and Their Physicochemical Properties, Crystal Growth \& Design, 9(6), 2009, 2950-2967.

[2] M.E. Aulton, Pharmaceutics: the science of dosage form design (Edinburgh, NY: Churchill Livingstone, 2002)

[3] F. Tian, H. Qu, A. Zimmermann, T. Munk, A.C. Jorgensen, J. Rantanen, Factors affecting crystallization of hydrates, Journal of Pharmacy and Pharmacology, 62, 2010, 1534-1546.

[4] A.V. Yadav, A.S. Shete, A.P. Dabke, P.V. Kulkarni, S.S. Sakhare, Co-crystals: A novel approach to modify physicochemical properties of active pharmaceutical ingredients, Indian Journal Pharmaceutical Sciences, 71(4), 2009, 359-370.

[5] K. Izutsu, T. Koide, N. Takata. Y. Ikeda, M. Ono, M. Inoue, T. Fukami, E. Yonemochi, Characterization and Quality Control of Pharmaceutical Cocrystals, Chemical and Pharmaceutical Bulletin, 64(10), 2016, 1421-1430.

[6] P.J. Barnes, Theophylline, American Journal of Respiratory and Critical Care Medicine, 188(8), 2013, 901-906,

[7] D. Komes, D. Horžić, A. Belščak , K.K. Ganič, A. Baljak, Determination of Caffeine Content in Tea and Maté Tea by Using Different Methods, Czech Journal of Food Sciences, 27, 2009, S213-S216.

[8] H.G.M. Edwards, E. Lawson, M. Matas, L. Shields, P. York, Metamorphosis of caffeine hydrate and anhydrous caffeine, Journal of the Chemical Society, Perkin Transactions 2, 1997, 1985-1990.

[9] E. Suihko, J. Ketolainen, A. Poso, M. Ahlgren, J. Gynther, P. Paronen, Dehydration of theophylline monohydrate a two step process, International Journal of Pharmaceutics, 158, 1997, 47-55.

[10] L. Takacs, The historical development of mechanochemistry, Chemical Society Reviews, 42, 7649-7659.

[11] A. Delori, T. Friscic, W. Jones, The role of mechanochemistry and supramolecular design in the development of pharmaceutical materials, CrystEngComm, 14, 2012, 2350-2362.

[12] M.M. Nolasco, A.M. Amado, P.J.A. Ribeiro-Claro, Computationally-Assisted Approach to the Vibrational Spectra of Molecular Crystals: Study of Hydrogen-Bonding and Pseudo- Polymorphism, ChemPhysChem, 7, 2006, 2150-2161.

[13] C. Liu, L. Dang, Y. Tong, H. Wei, Influence of Polymorphs on the Transformation Water Activity of Theophylline, Industrial \& Engineering Chemistry Research, 52, 2013, 14979-14983. 